\title{
Criptococose intestinal em um cão: relato de caso*
}

\section{Intestinal cryptococcosis in a dog: a case report}

\author{
Mariana Correia Oliveira, ${ }^{* *}$ Anieli Vidal Stocco, ${ }^{* * *}$ Stephanie Cardoso Silva, ${ }^{* * *}$ Carlos Augusto dos Santos Sousa, ${ }^{* * * *}$ \\ Rodrigo Mencalha Moreira, ${ }^{* * * *}$ Marcelo Abidu-Figueiredo******
}

\begin{abstract}
Resumo
A criptococose é uma micose sistêmica que em cães frequentemente está envolvida com lesões nos sistemas respiratório e nervoso central. O acometimento do sistema gastrointestinal por este fungo é considerada rara. O objetivo deste estudo é relatar um caso de criptococose intestinal em um cão, macho, da raça Boxer, de quatro anos, com histórico de êmese e diarreia sanguinolenta há cinco dias. Ao exame físico foi constatado dor abdominal e estrutura firme em região mesogástrica. Após realização de exame ultrassonográfico sugestivo de intussuscepção, realizou-se a celiotomia exploratória, e foi visualizada uma lesão nodular de $5 \mathrm{~cm}$ na parede do jejuno, retirada por enterectomia. O fragmento intestinal contendo a lesão foi encaminhado para análise histopatológica. Na macroscopia, a massa apresentava aspecto gelatinoso aos cortes, com superfície de corte compacta e tonalidade creme. No exame histopatológico, observou-se acentuada quantidade de estruturas leveduriformes, redondas ou ovoides, circundada por espessa cápsula, e discreto e difuso processo inflamatório mononuclear. A coloração pelo ácido periódico de Schiff (PAS) ajudou a confirmar a infecção pelo fungo do gênero Cryptococcus. Não foi possível dar continuidade ao caso, uma vez que o proprietário não retornou mais ao atendimento. Sugere-se que a porta de entrada da criptococose neste estudo, tenha sido através da ingestão da levedura desidratada, ou pela entrada do agente na extensa ulceração em jejuno, já que o mesmo pode ser encontrado em intestino delgado de cães saudáveis e de cães com enteropatia crônica. Conclui-se que, apesar da criptococose intestinal em cães ser considerada rara, recomenda-se a inclusão nos diagnósticos diferenciais de lesões que acometem os segmentos intestinais sem o envolvimento clínico de demais sistemas.
\end{abstract}

Palavras-chave: canino, intestino, micose, criptococose.

\section{Abstract}

Cryptococcosis is a systemic mycosis and is often associated with lesions in the respiratory and central nervous systems in dogs. However, the involvement of the gastrointestinal system in this fungal infection is rare. This study reports a case of intestinal cryptococcosis in a 4-year-old male Boxer dog with a history of emesis and bloody diarrhea for five days. His physical examination revealed abdominal pain and a firm structure in the mesogastric region. After an ultrasound examination suggested intussusception, exploratory celiotomy was performed, and a $5 \mathrm{~cm}$ mass was visualized on the wall of the jejunum, which was subsequently removed by an enterectomy and sent for histopathological analysis. Macroscopically, the mass presented a gelatinous aspect, with a compact cut surface and cream shade. On histopathological examination, multiple leveduriform structures, round or ovoid, surrounded by a thick capsule, and discrete, diffuse mononuclear inflammatory processes were observed. Schiff's periodic acid staining confirmed the infection with a fungus of the genus Cryptococcus. It was not possible to continue the investigation because the owner did not return to the service. It is suggested that the entry point of cryptococcosis was through the ingestion of dehydrated yeast or through extensive jejunal ulceration, as it can be found in the small intestine of both healthy dogs and those with chronic enteropathy. Therefore, although intestinal cryptococcosis in dogs is rare, it is recommended to include it as a differential diagnosis for gastrointestinal lesions without the clinical involvement of other systems.

Keywords: canine, intestine, mycosis, cryptococcosis

\footnotetext{
*Recebido em 10 de setembro de 2016 e aceito em 19 de outubro de 2017.

${ }^{* *}$ Discente do Programa de Pós-graduação em Medicina Veterinária (Patologia Animal), Universidade Federal Rural do Rio de Janeiro.

***Discente de Graduação em Medicina Veterinária/ Universidade Federal Rural do Rio de Janeiro.

****Discente do Programa Pós-Graduação em Biologia Animal / Universidade Federal Rural do Rio de Janeiro.

*****Docente da Faculdade de Medicina Veterinaria do Centro de Ensino Superior de Valença.

${ }^{* * * * * *}$ Docente do Laboratório de Ensino e Pesquisa em Morfologia de Animais Domésticos e Selvagens/ Departamento de Anatomia Animal e Humana/ Universidade Federal Rural do Rio de Janeiro.

Autor para correspondência: marceloabidu@gmail.com
} 


\section{Introdução}

A criptococose é uma doença causada pelo fungo leveduriforme do gênero Cryptococcus, que acomete seres humanos e muitas espécies animais (Sykes \& Malik, 2012).

A inalação de organismos suspensos no ar para o trato respiratório é considerada a principal porta de entrada dos organismos (Sykes \& Malik, 2012), porém a ingestão de leveduras desidratadas ou, mais raramente, inoculação cutânea são também consideradas como fontes de infecção (Martins et al., 2011).

Alguns fatores predisponentes como o estresse, doenças concomitantes ou a administração de corticosteroides podem permitir o desenvolvimento de doença clínica (Sykes \& Malik, 2012), entretanto já é sabido que o fungo Cryptococcus gattii também acomete indivíduos imunocompetentes, inclusive em caninos (Abreu et al., 2017).

A criptococose geralmente ocorre em cães com idade média de 2,2 anos, não existindo prevalência por sexo (Trivedi et al., 2011). A criptococose está mais frequentemente envolvida com lesões no sistema nervoso central e nos olhos (McGill et al., 2009) e menos frequentemente encontrado no sistema gastrointestinal e demais órgãos (Sykes \& Malik, 2012).

O fungo Cryptococcus spp. pode ser identificado por cultura de aspirados de tecidos, exsudato nasal, secreções traqueais, urina, fezes, citologia e histopatologia (Bivanco et al., 2006; Martins et al., 2008; Trivedi et al., 2011).

Devido à existência de poucos relatos na literatura sobre a criptococose em cão com o acometimento intestinal, objetivouse relatar um caso clínico em um cão jovem atendido em uma Clínica Veterinária no Estado do Rio de Janeiro.

\section{Relato de caso}

Relata-se um caso de um cão, macho, quatro anos de idade, da raça Boxer, não castrado, vacinação atualizada, domiciliado, com histórico de êmese e diarreia sanguinolenta há cinco dias. Ao exame físico foi constatado dor abdominal e estrutura firme em região mesogástrica com os demais parâmetros dentro da normalidade.

O animal foi submetido ao exame ultrassonográfico o qual apresentou imagens sugestivas de intussuscepção em segmento de jejuno. Foram realizados hemograma, (leucócitos- 39.200 células $/ \mathrm{ml}$ e proteínas totais- $4,1 \mathrm{~g} \%$ ) e perfil bioquímico renal e hepático que não apresentaram alterações. No mesmo dia foi realizada uma celiotomia exploratória confirmando a presença de intussuscepção envolvendo os segmentos de jejuno, e notada à presença de uma lesão nodular de aproximadamente cinco centímetros de diâmetro na parede do jejuno.

$\mathrm{O}$ animal foi submetido aos procedimentos de enterectomia e enteroanastomose e permaneceu internado durante dois dias. O tratamento instituído constou de ampicilina $25 \mathrm{mg} / \mathrm{kg} / \mathrm{TID}$, metronidazol $20 \mathrm{mg} / \mathrm{kg} / \mathrm{BID}$, ranitidina $2 \mathrm{mg} / \mathrm{kg} / \mathrm{TID}$, ondasetrona $0,22 \mathrm{mg} / \mathrm{kg} / \mathrm{TID}$, dipirona e escopolamina $25 \mathrm{mg} / \mathrm{kg} / \mathrm{TID}$. O fragmento intestinal contendo a lesão foi encaminhado para análise histopatológica. Na macroscopia, a lesão apresentava aspecto nodular gelatinoso aos cortes, com superfície compacta e tonalidade creme (Figura 1).

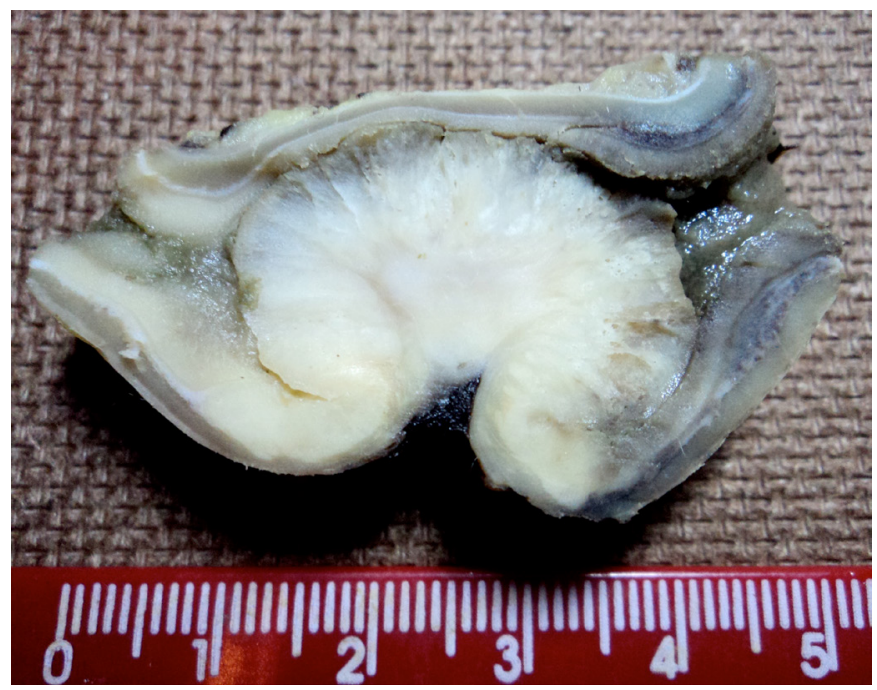

Figura 1: Corte sagital de lesão nodular encontrada no jejuno de um cão, com aproximadamente $5 \mathrm{~cm}$ de comprimento e $3 \mathrm{~cm}$ de largura, tonalidade creme, aspecto gelatinoso e consistência gelatinosa aos cortes.

No exame histopatológico, observou-se discreto e difuso processo inflamatório mononuclear composto por linfócitos, plasmócitos e macrófagos associados à acentuada quantidade de estruturas leveduriformes, redondas ou ovoides, circundada por espessa cápsula, conclusivos com infecção por fungos do gênero Cryptococcus. Verificou-se uma extensa ulceração se estendendo da camada mucosa à submucosa do órgão (Figura 2).

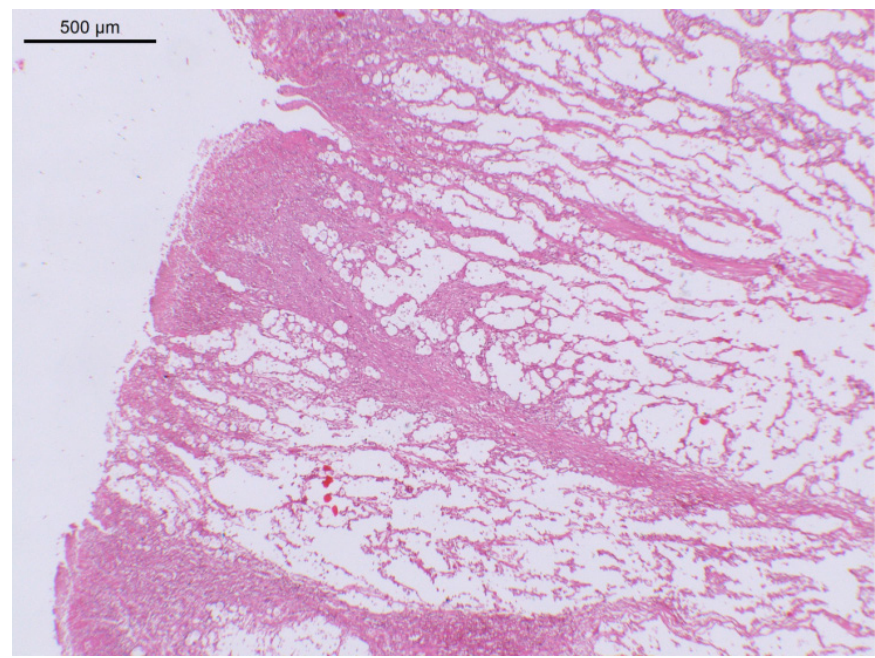

Figura 2: Corte histológico de lesão nodular encontrada no jejuno de um cão, evidenciando ulceração do epitélio e destruição de vilosidades, com acentuada presença de estruturas leveduriformes, redondas ou ovoides, compatíveis com fungo do gênero Cryptococcus. Coloração por HematoxilinaEosina. 


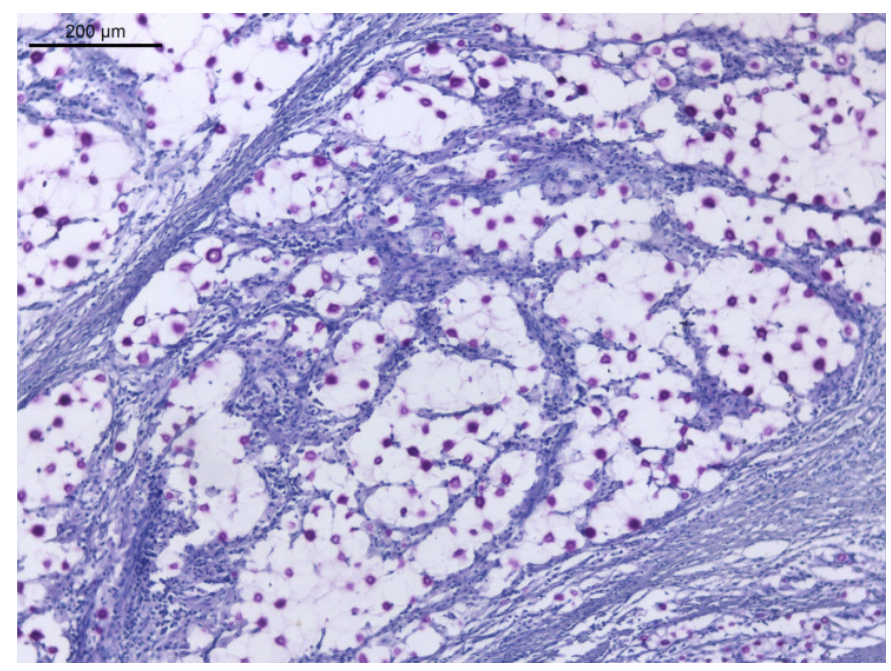

Figura 3: Corte histológico de lesão nodular encontrada no jejuno de um cão, evidenciando estruturas leveduriformes, redondas ou ovóides, em tonalidade púrpura-magenta, circundada por espessa cápsula sem afinidade tintorial, compatíveis com fungo do gênero Cryptococcus. Coloração pelo Ácido Periódico de Schiff (PAS).

Deste modo, foi prescrito itraconazol $10 \mathrm{mg} / \mathrm{kg} / \mathrm{SID}$, e, após dez dias de tratamento o animal retornou com melhora da hematoquezia, mas ainda com fezes pastosas. No exame ultrassonográfico foi observado linfonodo mesentérico medindo $8,1 \mathrm{~cm}$ de comprimento com aspecto grosseiro. Não foi possível dar continuidade ao caso, uma vez que o proprietário não retornou mais ao atendimento.

\section{Discussão e conclusão}

No presente relato, o diagnóstico histopatológico da massa no segmento de jejuno, permitiu a definição do diagnóstico conclusivo, concordando com Okagaki et al. (2010).

No presente estudo, o cão foi submetido ao exame ultrassonográfico, o qual revelou imagens sugestivas de intussuscepção em segmento de alça intestinal, concordando com o trabalho realizado por Duncan, Stephen \& Campbell (2006), onde um cão foi submetido à cirurgia de intussuscepção e o diagnóstico histopatológico revelou criptococose intestinal.

A semelhança com a intussuscepção no exame de imagem e com lesões neoplásicas à macroscopia pode dificultar o diagnóstico, portanto, alerta-se para à realização de exames complementares para alcançar o diagnóstico rápido e preciso e implementação da terapia.

\section{Referências}

ABREU, D.P.B.; MACHADO, C.H.; MAKITA, M.T.; BOTELHO, C.F.M.; OLIVEIRA, F.G.; VEIGA, C.C.P.; MARTINS, M.A.; BARONI, F.A. Intestinal Lesion in a Dog Due to Cryptococcus gattii Type VGII and Review of Published Cases of Canine Gastrointestinal Cryptococcosis. Mycopathologia, v. 182, n. 5-6, p. 597-602, 2017.

BIVANCO, F.C.; MACHADO, C.A.S.; MARTINS, E.L. Criptococose cutânea. Arquivos Médicos do ABC, v. 31, n. 2, p. 102-9, 2006.
Em estudo realizado por Malik et al. (1995), com 20 cães de idade média de dois anos, de ambos os sexos, apenas um cão apresentava criptococose intestinal com vômitos e linfadenite mesentérica, portanto a criptococose intestinal é considerada rara. O cão do presente relato apresentou sinais clínicos de criptococose intestinal incluindo letargia e está de acordo com a literatura consultada (Graves et al., 2005), emagrecimento progressivo, êmese e diarreia sanguinolenta.

A Criptococose gastrointestinal foi relatada no Brasil em uma cadela da raça Bulldog Inglês, de 18 meses com episódios de vômito, perda de peso progressivo, melena e prostração. $\mathrm{Na}$ ultrassonografia foi visualizada uma massa no intestino delgado que resultava em diminuição do lúmen. Após a realização da celiotomia exploratória, observou-se uma massa que media $18 \times 7 \mathrm{~cm}$. O exame microbiológico da massa resultou no isolado do gênero Cryptococcus gattii (Abreu et al., 2017).

A criptococose com envolvimento do trato gastrointestinal foi relatada em dois cães, sem o comprometimento em outro órgão (Malik et al., 1999), e sugere que o intestino possa ter sido o principal local de infecção nestes pacientes, pois é o que ocorre ocasionalmente em humanos (Daly et al., 1990).

Um estudo realizado por Suchodolski et al. (2008), avaliaram a prevalência de DNA fúngicos em intestino delgado de cães saudáveis e de cães com enteropatia crônica, nos Estados Unidos e na Europa, e nos resultados, três gêneros se destacaram na pesquisa, inclusive o fungo do gênero Cryptococcus. No presente relato, o cão acometido apresentava uma extensa ulceração em jejuno, se estendendo da camada mucosa à submucosa. Sugere-se, portanto, que a infecção possa ter se iniciado a partir de uma lesão prévia, o que serviu como porta de entrada para o agente fúngico em questão, ou ainda, que a ingestão da levedura desidratada tenha sido a porta de entrada para os organismos criptocócicos.

$\mathrm{Na}$ histopatologia, as leveduras apresentaram uma célula central, com presença de um núcleo, de forma ovoide ou esférica, circundada por uma cápsula espessa de polissacarídeo, que não se corou pela técnica de Hematoxilina-Eosina (Jubb \& Kennedy, 2016), e que ocasionalmente exibiam brotamento solitário (Okagaki et al., 2010), o que concorda ao observado na presente investigação. A coloração pelo ácido periódico de Schiff (PAS) permitiu evidenciar as estruturas leverudiformes e diferenciá-las de outras micoses sistêmicas.

Conclui-se que, apesar da criptococose intestinal em cães ser considerada rara, recomenda-se a inclusão nos diagnósticos diferenciais de lesões que acometem os segmentos intestinais sem o envolvimento clínico de demais sistemas.

DALY J.S.; PORTER, K.A.; CHONG, F.K.; ROBILLARD, R.J. Disseminated, nonmeningeal gastrointestinal cryptococcal infection in an HIVnegative patient. American Journal Gastroenterology, v. 85, p. 1421-1424, 1990.

DUNCAN, C.; STEPHEN, C.; CAMPBELL, J. Clinical characteristics and predictors of mortality for Cryptococcus gattii infection in dogs and cats of southwestern British Columbia. Canadian Veterinary Journal, v.47, p. 993-998, 2006.

GRAVES, T.K.; BARGER, A.M.; ADAMS, B.; KROCKENBERGER, M.B. Diagnosis of systemic cryptococcosis by fecal cytology in a dog. Veterinary Clinical Pathology, v. 34, n. 4, p. 409-412, 2005. 
KENNEDY, J. PALMER'S. Pathology of Domestic Animals. 6thed. Ontario, Canadá: Elsevier, 2016, p. 543-545.

MALIK, R.; DILL-MACKY, E.; MARTIN, P.; WIGNEY, D.I.; MUIR, D.B.; LOVE, D.N. Cryptococcosis in dogs: a retrospective study of 20 consecutive cases. Journal Medical Veterinary Mycology, v. 33, n. 5 , p. 291-297, 1995.

MALIK, R.; HUNT, G.B.; BELLENGER, C.R. Intra abdominal cryptococcosis in two dogs. Journal Small Animal Practical., v. 40, p. 387-391, 1999.

MARTINS, D.B.; BARBOSA, A.L.T., CAVALHEIRO, A.; LOPES, S.T.A.; SANTURIO, J.M.; SCHOSSLER, J.E.; MAZZANTI, A. Diagnóstico de criptococose canina pela citologia aspirativa por agulha fina. Ciência Rural, v. 38, p. 826-9, 2008.

MARTINS, D.B.; ZANETTE, R.A.; FRANÇA, R.T.; HOWES, F.; AZEVEDO, M.I.; BOTOON, S.A.; MAZZANTI, C.; LOPES, S.T.A.; SANTURIO, J.M. Massive cryptococcal disseminated infection in a immunocompetent cat. Veterinary Dermatology, v. 22, p. 232-234, 2011.

McGILL, S.; MALIK, R.; SAUL, N.; BEETSON, N.; SECOMBE, C.; ROBERTSON, I.; IRWIN, P. Cryptococcosis in domestic animals in Western Australia: a retrospective study from 1995-2006. Medical Mycology, v. 47, p. 625-639, 2009.
OKAGAKI, L.H.; STRAIN, A.K.; NIELSEN, J.N.; CHARLIER, C.; BALTES, N.J; CHRÉTIEN, F.; HEITMAN, J.; DROMER, F.; NIELSEN, K. Cryptococcal cell morphology affects host cell interactions and pathogenicity. Plos Pathogens. v. 6, n. 6, p. 115, 2010.

SUCHODOLSKI, J. S.; MORRIS, E.R.; ALLENSPACH, K..; JERGENS, A.E.; HARMOINEN, J. A.; WESTERMARCK, E.; STEINER, J. M. Prevalence and identification of fungal DNA in the small intestine of healthy dogs and dogs with chronic enteropathies. Veterinary Microbiology, v. 132, n. 3-4, p. 379-388, 2008.

SYKES, J.E.; MALIK, R. Cryptococcosis. In: Greene C.E., editor. Infectious disease of the dog and cat. 4th ed. Missouri: Elsevier; 2012, p. 621-634.

TRIVEDI, S.R.; SYKES, J.E.; CANNON, M.S.; WISNER, E.R.; MEYER, W.; STURGES, B.K.; DICKINSON, P.J.; JOHNSON, L.R. Clinical features and epidemiology of cryptococcosis in cats and dogs in California: 93 cases (1988-2010). JAVMA. V. 239, n.3, p. 357-369, 2011. 